\title{
Relating Global and Local Connectome Changes to Dementia and Targeted Gene Expression in Alzheimer's Disease
}

\author{
Samar S. M. Elsheikh ${ }^{1,2}$, Emile R. Chimusa ${ }^{3}$, Alzheimer's Disease Neuroimaging Initiative, \\ Nicola J. Mulder ${ }^{2 *}$ and Alessandro Crimi ${ }^{4,5,6}$ \\ 1 Pharmacogenetic Research Clinic, Centre for Addiction and Mental Health, Campbell Family Mental Health Research \\ Institute, Toronto, ON, Canada, ${ }^{2}$ Computational Biology Division, Department of Integrative Biomedical Sciences, Faculty of \\ Health Sciences, Institute of Infectious Disease and Molecular Medicine, University of Cape Town, Cape Town, South Africa, \\ ${ }^{3}$ Division of Human Genetics, Department of Pathology, Faculty of Health Sciences, Institute of Infectious Disease and \\ Molecular Medicine, University of Cape Town, Cape Town, South Africa, ${ }^{4}$ Computer Vision Group, Sano Centre for \\ Computational Medicine, Kraków, Poland, ${ }^{5}$ Institute for Neuropathology, University Hospital of Zurich, Zurich, Switzerland, \\ ${ }^{6}$ Department of Mathematics, African Institute for Mathematical Sciences, Cape Coast, Ghana
}

\section{OPEN ACCESS}

Edited by:

Jussi Tohka,

University of Eastern Finland, Finland

Reviewed by:

Lei Du,

Northwestern Polytechnical University,

China

Ibai Diez,

Massachusetts General Hospital and Harvard Medical School,

United States

*Correspondence:

Nicola J. Mulder nicola.mulder@uct.ac.za

Specialty section:

This article was submitted to Brain Imaging and Stimulation,

a section of the journal

Frontiers in Human Neuroscience

Received: 19 August 2021 Accepted: 25 November 2021 Published: 17 December 2021

Citation:

Elsheikh SSM, Chimusa ER Alzheimer's Disease Neuroimaging Initiative, Mulder NJ and Crimi A (2021) Relating Global and Local

Connectome Changes to Dementia and Targeted Gene Expression in

Alzheimer's Disease.

Front. Hum. Neurosci. 15:761424. doi: 10.3389/fnhum.2021.761424
Networks are present in many aspects of our lives, and networks in neuroscience have recently gained much attention leading to novel representations of brain connectivity. The integration of neuroimaging characteristics and genetics data allows a better understanding of the effects of the gene expression on brain structural and functional connections. The current work uses whole-brain tractography in a longitudinal setting, and by measuring the brain structural connectivity changes studies the neurodegeneration of Alzheimer's disease. This is accomplished by examining the effect of targeted genetic risk factors on the most common local and global brain connectivity measures. Furthermore, we examined the extent to which Clinical Dementia Rating relates to brain connections longitudinally, as well as to gene expression. For instance, here we show that the expression of PLAU gene increases the change over time in betweenness centrality related to the fusiform gyrus. We also show that the betweenness centrality metric impact dementia-related changes in distinct brain regions. Our findings provide insights into the complex longitudinal interplay between genetics and brain characteristics and highlight the role of Alzheimer's genetic risk factors in the estimation of regional brain connectivity alterations.

Keywords: Alzheimer's disease, connectome, gene expression, connectivity metrics, diffusion-weighted imaging, dementia, PLAU, MRI

\section{INTRODUCTION}

The advancement in technologies and the integration of genetic and neuroimaging datasets have taken Alzheimer's research steps further, and produced detailed descriptions of molecular and brain aspects of the disease (Shaw et al., 2007). Previous studies have utilized the connectome (Hagmann et al., 2008) to study different brain diseases through associating genetic variants to brain connectivity (Thompson et al., 2014). A structural connectome is a representation of the brain as a network of distinct brain regions (nodes) and their structural connections (edges), calculated as the number of anatomical fibers. Those anatomical fibers are generally obtained by diffusion-weighted imaging (DWI) (Alexander et al., 2007). DWI is the most commonly 
used in-vivo method for mapping and characterizing the diffusion of water molecules in three-dimensions, as a function of the location, and ultimately to construct the structural connectome. The connectome representation of the brain allows measuring of important properties, such as the ability of the brain to form separated sub-networks (network segregation), or the measure of a network dispersion (i.e., how segregated subnetworks are connected network integration) (Deco et al., 2015). Given such measures of the brain, it is also possible to represent each individual brain as single scalar metrics which summarize peculiar properties of the network's segregation and integration (Rubinov and Sporns, 2010), and calculate what is known as global connectivity metrics. Moreover, the connectome can also be used to quantify local properties of the brain (i.e., properties at specific nodes/areas). These measures (i.e., global and local connectivity metrics) can reflect neurodegeneration in the sense that neuronal apoptosis (i.e., programmed cell death) can be represented as a reduction in structural connectivity (Douaud et al., 2007; Elsheikh et al., 2020b).

There are many factors which may affect susceptibility to Alzheimer's disease (AD) and various ways to measure the disease status. However, there is no single factor which can be used to predict the disease risk sufficiently (Barnes and Yaffe, 2011). Genetics is believed to be the most common risk factor in AD development (Gatz et al., 1997). Genetic variants located in about 20 genes have been reported to affect the disease through many cell-type specific biological functions (Gaiteri et al., 2016). Genome-Wide Associations Studies (GWAS), also highlighted dozens of multi-scale genetic variations associated with AD risk (Lambert et al., 2013; Escott-Price et al., 2014; Elsheikh et al., 2020b). From the early stages of studying the disease, the well known genetic risk factors of $\mathrm{AD}$ were found to lie within the coding genes of proteins involved in amyloid$\beta(A \beta)$ processing. These include the Apolipoprotein $\mathrm{E}$ gene $(A p o E)$ which increases the risk of developing AD (Corder et al., 1993), the Amyloid precursor protein (APP) (Goate et al., 1991), presenilin-1 (PSEN1), and presenilin-2 (PSEN2) (Levy-Lahad et al., 1995; Rogaev et al., 1995).

Early work demonstrated that ApoE-4 carriers have an accelerated age-related loss of global brain inter-connectivity in AD subjects (Brown et al., 2011), and topological alterations of both structural and functional brain networks are present even in healthy subjects carrying the ApoE gene (Chen et al., 2015). A meta-analysis study also showed the impact of APOE, phosphatidylinositol binding clathrin assembly protein (PICALM), clusterin (CLU), and bridging integrator 1 (BIN1) gene expression on resting state functional connectivity in $\mathrm{AD}$ patients (Chiesa et al., 2017). Going beyond the ApoE gene, Jahanshad et al. (2013) used a dataset from the Alzheimer's Disease Neuroimaging Initiative (ADNI) to carry out a GWAS of brain connectivity measures and found an associated variant in F-spondin (SPON1), previously known for its association with dementia severity. A more recent study has also used the ADNI dataset to conduct a GWAS on the longitudinal brain connectivity, and provided an evidence of association between some single nucleotide polymorphisms (SNPs) in the CDH18 gene and Louvain modularity (a measure of network segregation) (Elsheikh et al., 2020b). A landmark study identified reproducible gene expression signatures related to functional brain connectivity (Hawrylycz et al., 2015). Cortical atrophy related to Alzheimer's was associated with regional expression levels of MAPT from the Allen brain atlas (Grothe et al., 2018). Subsequently, further characterization of longitudinal misfolded protein spreading was also associated with regional expression levels of CLU (Sepulcre et al., 2018). a recent review summarized these findings and other connectomics related studies (Yu et al., 2021).

$\mathrm{AD}$ is a common dementia-related illness; in the elderly, $\mathrm{AD}$ represents the most progressive and common form of dementia. Accordingly, incorporating and assessing dementia severity when studying $\mathrm{AD}$ provides more insights into the disease progression from a clinical point of view. A reliable global rating of dementia severity is the Clinical Dementia Rating (CDR) (Morris et al., 1997), which represents a series of specific evaluations for memory, orientation, judgment and problem solving, community affairs, home and hobbies (intellectual interests maintained at home), and personal care. The ultimate CDR measure is an ordinal scale which rates the severity of dementia symptoms, it uses the values $0,0.5,1,2$, and 3 , to represent none, very mild, mild, moderate, and severe, respectively.

In this paper, we integrated different aspects of Alzheimer's Disease, including longitudinal measures of CDR, global and local connectivity as well as gene expression extracted from blood samples of $\mathrm{AD}$ patients and controls. For global connectivity, we utilized the most commonly used metrics of network segregation (i.e., Louvain modularity and transitivity) and integration (i.e., characteristic path length and global efficiency). Path length was used in a plethora of studies as a biomarker to study the complex brain connectivity in schizophrenia (van den Heuvel et al., 2010), and the local efficiency and characteristic path lengths that were used to study the structural organization of the brain network in autism (Rudie et al., 2013). For a detailed review on work using such metrics see (Griffa et al., 2013). A brief description of the global connectivity metrics we used here, 1) Louvain modularity is a community (cluster) detection method, which iteratively transforms the network into a set of communities, 2) transitivity quantifies the segregation of a network by normalizing the fraction of triangles around an individual node, 3) characteristic path length is the average shortest path, 4) the global efficiency is the inverse of characteristic path length. For the local connectivity features, we used one measure to represent node's segregation, integration and centrality. The local connectivity metrics of a network represent large-scale organization which in turn may be used to represent well functioning cognitive functions (Cohen and D’Esposito, 2016). Measures of centrality (e.g., degree and betweenness centrality) usually measure similar features in a network (such as the amount or weight of connections that a node has), and hence, can be highly related to each other. Indeed, it has been shown that many of them can lead back to the degree centrality at a node (Rubinov, 2016). Therefore, along with using local connectivity measures, we computed their correlation with the nodal degree metric-the simplest feature we can extract from a graph node. 
Our work aimed to answer questions such as are there brain connectivity metrics that discriminate changes longitudinally in $\mathrm{AD}$ patients compared to healthy control subjects? Is there a most representative metric, or a redundancy in the chosen metrics? Is there a correlation between the metrics used and expression of known $\mathrm{AD}$-related genes? Is there a correlation between connectivity metrics and clinical ratings?

We address these questions considering the global brain by using global connectivity, and also considering specific brain regions using the local connectivity metrics. We regressed the absolute changes in connectivity metrics on gene expression, studied the association between the longitudinal changes of connectivity and CDR scores, and performed a ridge regression between gene expression, the changes in CDR scores and brain connectivity.

Using a dataset from ADNI (http://adni.loni.usc.edu/) this paper presents an integrated association study of specific $\mathrm{AD}$ risk genes, dementia scores and structural connectome characteristics. Specifically, we used the longitudinal case-control dataset to examine the association of known $\mathrm{AD}$ risk-gene expression with the change in local and global connectivity metrics. Furthermore, we tested the longitudinal effect of brain connectivity on the difference (i.e., change over time) of CDR scores through a multivariate analysis to study the longitudinal effect of gene expression and connectome changes on CDR change. The identification of advanced imaging and genetic biomarkers with regional effect can be put into clinical practice, specially, the treatment of specific regions of the brain though targeting circuitry affected by plaques (Canter et al., 2019).

\section{MATERIALS AND METHODS}

\section{Data Description}

We used two sets of data from ADNI, which are available at adni.loni.usc.edu. The experiments have been conducted on the publicly available datasets described below, for which ethical approval has already been granted, and data acquisition has been conducted according to the Helsinki II regulations. Additionally, we received ethics approval from the Faculty of Health Sciences Human Research Ethics Committee at the University of Cape Town. The populations are matched by age, and the mean ages are, respectively, $76.5 \pm 7.4$ for $\mathrm{AD}$ patients, and $77.0 \pm 5.1$ years for healthy subjects. To fulfill our objectives, unless otherwise specified, we merged neuroimaging, gene expression and CDR datasets for all the participants, who have these three types of data available, at two-time points. We considered follow-up imaging and CDR acquisition 1 year later than the baseline visit. All those constraints drastically reduced the number of available samples, yielding a total of 51 participants, $15 \mathrm{AD}$ patients, and 36 healthy elderly subject. Recent studies (Richiardi et al., 2015) focused on genetics and MRI data also used similar sample sizes. Therefore, despite this limitation this kind of study offers novel insights combining all those multimodal data generally not reached by purely imaging or non-longitudinal studies.

\section{Imaging Data}

For the imaging, we obtained the DWI volumes at two time points, the baseline and follow-up visits, with 1 year in between. Along with the DWI, we used the T1-weighted images which were acquired using a GE Signa scanner 3T (General Electric, Milwaukee, WI, USA). The T1-weighted scans were obtained with voxel size $=1.2 \times 1.0 \times 1.0 \mathrm{~mm}^{3} \mathrm{TR}=6.984 \mathrm{~ms}$; $\mathrm{TE}=$ $2.848 \mathrm{~ms}$; flip angle $=11^{\circ}$, while DWI was obtained with voxel size $=1.4 \times 1.4 \times 2.7 \mathrm{~mm}^{3}$, scan time $=9 \mathrm{~min}$, and 46 volumes (5 T2-weighted images with no diffusion sensitization b0 and 41 diffusion-weighted images $b=1000 \mathrm{~s} / \mathrm{mm}^{2}$ ).

\section{Pre-processing of Imaging Data}

Each DWI and T1 volume had been pre-processed performing eddy current correction and skull stripping. DWI and T1 volumes were already co-registered, and the atlas was further linearly registered to them according to 12 degrees of freedom. We used the same T1 reference to get the information needed to compute the partial volume effect from the tissue segmentation by using the FMRIB Software Library (FSL). More specifically, we used FMRIB's Automated Segmentation Tool (FAST) to obtain 3 masks further used as anatomical constraints for the tractography. Linear registration was used as although atrophy was visible mostly in the cingulum, it was not case for the overall cortex.

\section{Genetic Data Acquisition}

We used the Affymetrix Human Genome U219 Array profiled expression dataset from ADNI. The RNA was obtained from blood samples and normalized before hybridization to the array plates. Partek Genomic Suite 6.6 and Affymetrix Expression Console were used to check the quality of expression and hybridization (Saykin et al., 2015). The expression values were normalized using the Robust Multi-chip Average (Irizarry et al., 2003), after which the probe sets were mapped according to the human genome (hg19). Further quality control steps were performed by checking the gender using specific gene expression, and predicting the SNPs from the expression data (Vawter et al., 2004; Schadt et al., 2012). Although it is more useful to extract the gene expression profiles from the brain, we used the gene expression extracted from blood samples, as provided by ADNI. Blood gives a general idea of what is happening in the body, and can detect differences in gene expression. Moreover, blood samples are easy to obtain and are noninvasive. Nevertheless, we verified that the investigated genes are expressed in the brain parenchyma through the Allen gene expression atlas https:// human.brain-map.org/ish/search and Protein Atlas https://www. proteinatlas.org. In this work, we targeted specific genes which have been reported to affect the susceptibility of AD. We used the BioMart software from Ensembl to choose those genes by specifying the phenotype as AD (Smedley et al., 2015). We obtained a total of 17 unique gene names and retrieved a total of 65 probe sets from the genetic dataset.

\section{Clinical Dementia Rating}

The Clinical Dementia Rating (CDR) score is an ordinal scale used to rate the condition of dementia symptoms. It ranges from 
0 to 3 , and is defined by five values: $0,0.5,1,2$, and 3 , ordered by severity (smaller values are less severe), these values stand for none, very mild, mild, moderate and severe, respectively. The scores evaluate the cognitive state and functionality of participants. Here, we used the main six scores of CDR; memory, orientation, judgement and problem solving, community affairs, home and hobbies, and personal care. Besides these, we used a global score, calculated as the sum of the six scores. We obtained the CDR scores at two time points in accordance with the connectivity metrics time points.

\section{Connectome Construction}

We generated tractographies by processing DWI data with Dipy (Garyfallidis et al., 2014), a Python library. More specifically, we used the constant solid angle model (Aganj et al., 2010) and Euler Delta Crossings (Garyfallidis et al., 2014) deterministic algorithm. We stemmed from 2,000,000 seed-points, and as stopping condition we used the anatomically-constrained tractography approach based on partial volume effect (Smith et al., 2012). We also discarded all fibers with sharp angle (larger than $75^{\circ}$ ) or those with length $<30 \mathrm{~mm}$.

To construct the connectome, we assigned a binary representation in the form of a matrix whenever connections were present between two Automated anatomical labeling (AAL) atlas (Tzourio-Mazoyer et al., 2002) regions, for any pair of regions. Though the AAL atlas has been criticized for functional connectivity studies (Gordon et al., 2014), it has been useful in providing insights in neuroscience and physiology, and is believed to be sufficient for our case study limited to structural data and large brain regions (Gordon et al., 2014).

\section{Global and Local Connectivity Metrics}

To quantify the overall efficiency and integrity of the brain, we extracted global measures of connectivity from the connectome, represented here in four values of network integration and segregation. Specifically, we used two network integration metrics 1) global efficiency $(E)$, and 2) weighted characteristic path length $(L)$. Both are used to measure the efficiency at which information is circulated around a network. On the other hand, we used; 1) Louvain modularity (Q), and, 2) transitivity $(T)$ to measure the segregation of the brain which is defined as the capability of the network to shape sub-communities which are loosely connected to one another while forming a densely connected sub-network within communities (Rubinov and Sporns, 2010; Deco et al., 2015).

Suppose that $n$ is the number of nodes in the network, $N$ is the set of all nodes, the link $(i, j)$ connects node $i$ with node $j$ and $a_{i j}$ define the connection status between node $i$ and $j$, such that $a_{i j}=1$ if the link $(i, j)$ exist, and $a_{i j}=0$ otherwise. We define the global connectivity metrics as; $E=$ $\frac{1}{n(n-1)} \sum_{i \in N} \sum_{j \in N, j \neq i} d_{i j}^{-1}$, where, $d_{i j}=\sum_{a_{u v} \in g_{i \leftrightarrow j}} a_{u v}$, is the shortest path length between node $i$ and $j$, and $g_{i \leftrightarrow j}$ is the geodesic between $i$ and $j . L=\frac{1}{n(n-1)} \sum_{i \in N} \sum_{j \in N, j \neq i} d_{i j} . Q=$ $\frac{1}{l} \sum_{i j \in N}\left[a_{i j}-\frac{k_{i} k_{j}}{l}\right] \delta\left(c_{i}, c_{j}\right)$, where $l=\sum_{i, j \in N} a_{i j}, m_{i}$ and $m_{j}$ are the modules containing node $i$ and $j$, respectively, and $\delta\left(c_{i}, c_{j}\right)=$
1 if $c_{i}=c_{j}$ and 0 otherwise. $T=\frac{\sum_{i \in N} 2 t_{i}}{\sum_{i \in N} k_{i}\left(k_{i}-1\right)}$, where $t_{i}=$ $\frac{1}{2} \sum_{j, h \in N}\left(a_{i j} a_{i h} a_{j h}\right)$ is the number of triangles around node $i$.

Moreover, using the AAL atlas, we constructed the following local brain network metrics at each region or node. We used the local efficiency $\left(E_{l o c, i}\right)$, clustering coefficient $\left(C_{i}\right)$ and betweenness centrality $\left(b_{i}\right)$ at each node to quantify the local connectivity. Local efficiency and clustering coefficient measure the presence of well-connected clusters around the node, and they are highly correlated to each other. The betweenness centrality is the number of shortest paths which pass through the node, and measures the effect of the node on the overall flow of information in the network (Rubinov and Sporns, 2010). The local connectivity metrics used in this work, for a single node $i$, are defined as follows;

$$
E_{l o c, i}=\frac{\sum_{j, h \in N, j \neq i} a_{i j} a_{i h}\left[d_{j h}\left(N_{i}\right)\right]^{-1}}{k_{i}\left(k_{i}-1\right)}, \text { where, } d_{j h}\left(N_{i}\right) \text {, is the length }
$$
of the shortest path between node $j$ and $h$-as defined in Equation, and contains only neighbors of h $C_{i}=\frac{2 t_{i}}{k_{i}\left(k_{i}-1\right)} \cdot b_{i}=$ $\frac{1}{(n-1)(n-2)} \sum_{h, j \in N, h \neq j, h \neq i, i \neq j} \frac{\rho_{h j}(i)}{\rho_{h j}}$, where $\rho_{h j}(i)$ is the weights of shortest path between $h$ and $j$ that passes through $i$.

\section{Statistical Analysis}

We used different statistical methods as described below, and for the multiple testing we relied on the Bonferroni correction (Narum, 2006; White et al., 2019). Where applicable, the thresholds were obtained by dividing 0.05 by the number of independent tests. We stated the corrected threshold value and the number of independent tests in the caption of each table in the Results section.

\section{Quantifying the Change in CDR and Connectivity Metrics}

To determine the longitudinal change in CDR, local and global connectivity metrics, we calculated the absolute difference between the first visit (the baseline visit) and the first visit after 12 months (the follow-up visit). Unless stated otherwise, this is the primary way we used to quantifying the longitudinal change in this analysis.

\section{Estimation of Gene Expression From Multiple Probe Sets}

Different probe set expression values were present for each gene in the data. To estimate a representative gene expression out of the probe set expression, we conducted a non-parametric Mann-Whitney $U$-test to evaluate whether the expression in $\mathrm{AD}$ was different from those of healthy elderlies. For each gene, we selected the probe set expression that has the lowest Mann-Whitney $U p$-value. In this way, we selected the most differentially expressed probe sets in our data and considered those for the remaining analysis.

\section{Spearman's Rank Correlation Coefficient}

To test the statistical significance of pair-wise undirected relationships, we used the Spearman's rank correlation coefficient $(\rho)$. The Spearman coefficient is a non-parametric method which ranks pairs of measurements and assesses their monotonic relationship. We report here the coefficient $\rho$ along with 

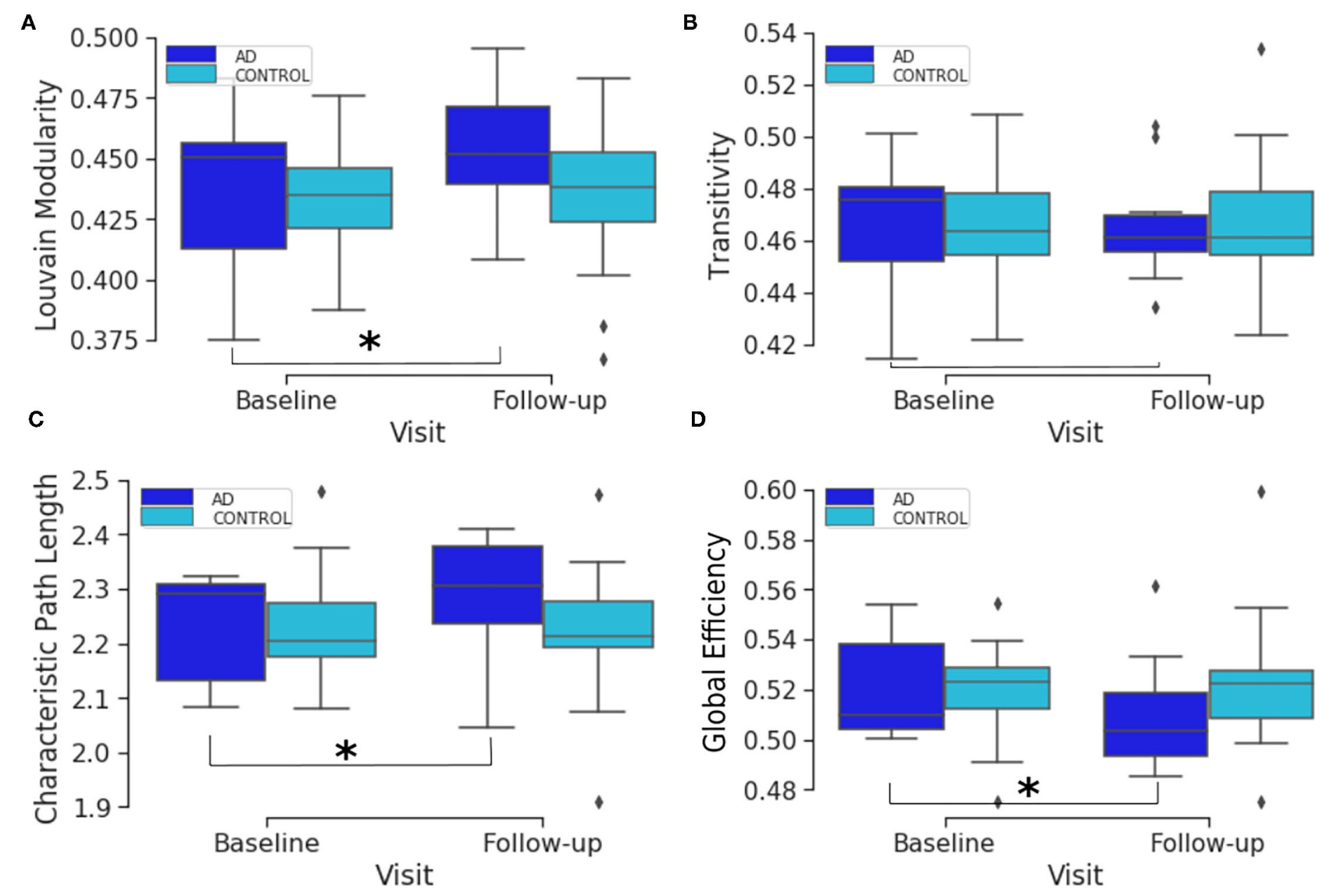

FIGURE 1 | Box plots of the distribution of brain segregation and integration global connectivity metrics comparing the two time points. The plots compare the baseline and follow-up distributions for AD and healthy elderly subjects for Louvain modularity (A), transitivity (B), characteristic path length (C) and global efficiency (D). The asterisk denotes that there is a significant change from baseline to the follow-up visit.
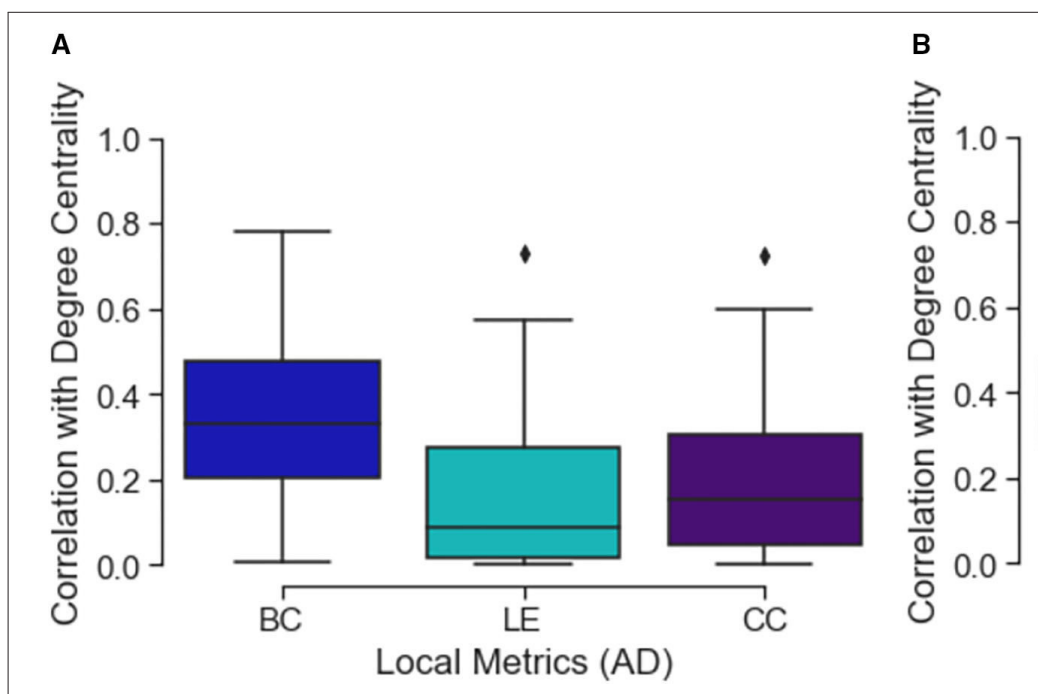

FIGURE 2 | The figure shows three box plots, respectively, (A) for the AD and (B) control subjects. Each boxplot represents the correlation values between the degree value of a node and the related local efficiency (LE), betweenness centrality (BC) and clustering coefficient (CC). Values close to 0 represent low correlation while those close to 1 show a high correlation. 
the corresponding $p$-value to evaluate the significance of the relationship. A $\rho$ of \pm 1 indicates a very strong relationship, while $\rho=0$ means there is no relationship.

\section{Quantile Regression}

To model the directed relationship between two variables, we used the quantile regression model (Koenker and Hallock, 2001). This model is used as an alternative to the linear regression model when the linear regression assumptions are not met. This fact allows the response and predictor variables to have a non-symmetric distribution. The quantile regression model estimates the conditional median of the dependent variable given the independent variables. Besides, it can be used to estimate any conditional quantile; and is therefore robust to outliers. Accordingly, in this work we used the quantile regression to model the directed relationship between two variables. As an alternative to the linear regression, we chose the $50^{\text {th }}$ percentile (the median) as our quantile and estimated the conditional median (rather than the conditional mean in case of the ordinary linear regression) of the dependent variable across given values of the independent variable.

\section{Ridge Regression}

For estimating the relationship between more than two variables, we used ridge regression (Hoerl and Kennard, 1970). The basic idea behind this model is that it solves the least square function penalizing it using the $l_{2}$ norm regularization. More specifically, the ridge regression minimizes the following objective function:

$\|y-X \beta\|_{2}^{2}+\alpha\|\beta\|_{2}^{2}$, i.e., $\beta^{\text {Ridge }}=\underset{\beta \in R}{\operatorname{argmin}}\|y-X \beta\|_{2}^{2}+$ $\alpha\|\beta\|_{2}^{2}$, where $y$ is the dependent (or response) variable, $X$ is the

TABLE 1 | Mann-Whitney $U$-test top results for the difference between AD and controls in probe set expression.

\begin{tabular}{|c|c|c|c|}
\hline \multirow[t]{2}{*}{ Gene } & \multicolumn{3}{|c|}{ Top results } \\
\hline & Chromosome & Probe set id & $p$-value \\
\hline APBB2 & 4 & 11734823_a_at & 0.02575 \\
\hline MPO & 17 & 11727442_at & 0.38631 \\
\hline$A P P$ & 21 & 11762804_x_at & 0.01396 \\
\hline$A C E$ & 17 & 11752871_a_at & 0.24478 \\
\hline$P L A U$ & 10 & 11717154_a_at & 0.01396 \\
\hline PAXIP1 & 7 & 11755176_a_at & 0.45499 \\
\hline HFE & 6 & 11736346_a_at & 0.11881 \\
\hline SORL 1 & 11 & 11743129_at & 0.10912 \\
\hline$A 2 M$ & 12 & 11715363_a_at & 0.28592 \\
\hline NOS3 & 7 & 11725467_a_at & 0.04261 \\
\hline$B L M H$ & 17 & 11757556_s_at & 0.09356 \\
\hline ADAM10 & 15 & 11751180_a_at & 0.14278 \\
\hline PLD3 & 19 & 11715382_x_at & 0.17304 \\
\hline$A p o E$ & 19 & 11744068_x_at & 0.05962 \\
\hline PSEN1 & 14 & 11718678_a_at & 0.29453 \\
\hline PSEN2 & 1 & 11723674_x_at & 0.04862 \\
\hline$A B C A 7$ & 19 & 11755091_a_at & 0.45499 \\
\hline
\end{tabular}

independent variable (feature, or predictor), $\beta$ is the ordinary least square coefficient (or, the slope), $\alpha$ is the regularization parameter, $\beta^{\text {Ridge }}$ is the ridge regression coefficient, argmin is the argument of minimum and it is responsible for making the function attain the minimum and is $L_{2}(v)=\|v\|_{2}$ which represents the L2 norm function (Strang, 1993). For example, the CDR scores are the response variables; and brain connectivity features or gene expressions are the predictor variables. We normalized the predictors to get a more robust estimation of our parameters.

\section{Software}

We used python 3.7.1 for this work; our code has been made available under the MIT License https://choosealicense.com/ licenses/mit/, and is accessible at https://github.com/elssam/ RGLCG.

\section{RESULTS}

\section{Descriptive Statistics}

Initially, we used descriptive statistics plots to visualize the data for the two populations; the $\mathrm{AD}$ and matched control subjects. To facilitate the integrated analysis, we looked into the different sets of data individually to have a better understanding of the underlying statistical distribution, and chose the best analysis methods accordingly. Firstly, we plotted the global and local connectivity metrics in a way that illustrates the longitudinal change between the two visits (i.e., baseline and follow-up visits). The follow-up visit was 1 year after the baseline screening. The global connectivity metric box plots show the baseline and follow-up distributions for both $\mathrm{AD}$ and healthy elderly for transitivity, Louvain modularity, characteristic path length and global efficiency (Figure 1). Figure 1 shows that the global longitudinal changes in connectome metrics are statistically significant among the $\mathrm{AD}$ subjects and not mere artifacts, but not within the control population which has non significant changes. In other words, comparing the two groups ( $\mathrm{AD}$ and healthy elderly) in terms of the change in global connectivity metrics overtime, the only significant differences found between baseline and follow-up were within the AD group. These were found in the characteristic path length ( $p$-value 0.0057), global efficiency ( $p$-value 0.0033$)$, and Louvain modularity ( $p$-value 0.0086 ). The test used here was the Wilcoxon signed rank test for paired samples with a threshold of $\frac{0.05}{4}=0.0125$, where the division by 4 is due to the multiple hypothesis correction.

Supplementary Figures S1-S3 show the distribution of the absolute differences between the two visits in local efficiency, clustering coefficient and betweenness centrality connectivity metrics, respectively, at each of the AAL brain regions. These figures show that the amount of change in local connectivity metrics varies across the $\mathrm{AAL}$ regions. A list of the brain atlas region names and abbreviations is available in Supplementary Table S1. Moreover, in Supplementary Figure S4, we show the scatter and violin plots of the six CDR scores, at the baseline and follow-up. The CDR scores are 
TABLE 2 | Top results of spearman associations between AD gene expression and local connectivity metrics.

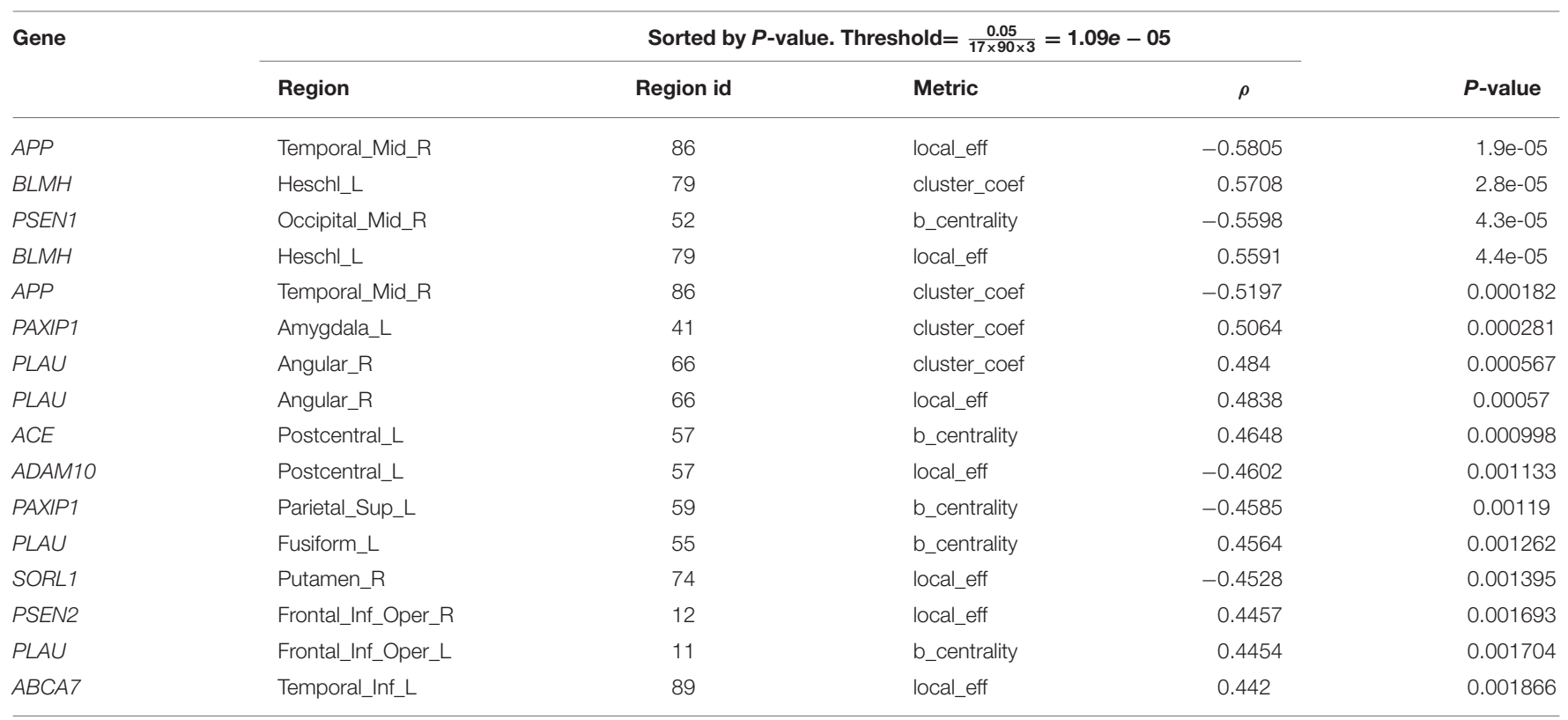

explained in the Materials and Methods section. As such, both global and local connectivity metrics show non-symmetric distribution in the baseline, followup and absolute change between them (see Figure 1 and Supplementary Figures S1-S3). Therefore, we will use non-parametric models and statistical tests in the following analysis.

Comparing the three local connectivity features to each other using Spearman correlation, as shown in Figure 3, we observe a high redundancy between local efficiency and cluster coefficients, assuming they are computed at the same time point. Moreover, we analyzed the relationship between the above-mentioned connectivity metrics and other related measures (e.g., degree centrality). As shown in Figure 2, the correlation between nodal degree and betweenness centrality appears to be low, whereas there is a negligible correlation between degree centrality and both local efficiency and clustering coefficient.

\section{Gene Expression}

We derived a list of $17 \mathrm{AD}$ risk factor genes from BioMart, and retrieved 56 related probes sets from ADNI data. We performed a Mann-Whitney $U$-test which aims at testing whether a specific probe set's expression is different between $\mathrm{AD}$ and healthy controls. For each gene, we chose the probe set that has the lowest $p$-value. Table 1 reports the selected probe set (with the minimum $p$-value) at each gene. It is worth mentioning that we are using probe sets, rather than single probes. These probe sets represent different parts of the transcripts rather than representing single alternative splicing of the gene, and are normalized across probes for each gene. Expression values were normalized using the robust multi-chip average method in the Affymetrix U219 array, which consists of a total number of 49,293 probe sets and 530,467 probes, as explained in the Genetic Data Acquisition section.
After estimating the expression of the 17 genes, as explained in the Materials and Methods, we plotted a heatmap of the gene expression profiles shown in Supplementary Figure S5. Some of the genes appear to be highly expressed (e.g., SORL1 and PSEN1), while others show very low expression (e.g., $H F E$ and $A C E$ ). To avoid double-dipping in estimating the effect size of gene expression, the subsequent analysis will not depend on disease status (i.e., $\mathrm{AD}$ and control), but rather, on quantitative measurements (e.g., local and global connectivity metrics) derived from the whole sample.

\section{Association Analysis}

We studied the undirected associations of the 17 gene expression values with the longitudinal change in global and local brain connectivity, as well as the associations with longitudinal CDR and connectivity changes. Firstly, we performed an association analysis of gene expression with the connectivity changes locally, at each AAL brain region. In Table 2 we show the top results reported along with the Spearman correlation co-efficient. The APP gene $(\rho=-0.58, p$ value $=1.9 \mathrm{e}-05)$ and $B L M H(\rho=0.57, p=2.8 \mathrm{e}-05)$ are the top genes in the list, and although they both did not hit the significance threshold, they show potential association with the change in local efficiency at the right middle temporal gyrus (Temporal_Mid_R AAL region) and clustering coefficient at the left Heschl gyrus (Heschl_L), respectively. Supplementary Figure S6 shows the scatter plots related to these significant associations.

In Table 2, there is a similar pattern observed in association results between the regional clustering coefficient and local efficiency, e.g., both metrics are associated with $B L M H$ at the left Heschl gyrus (Heschl_L), APP at the right middle temporal gyrus (Temporal_Mid_R) and PLAU at the right angular gyrus (Angular_R). We interpret this by the 


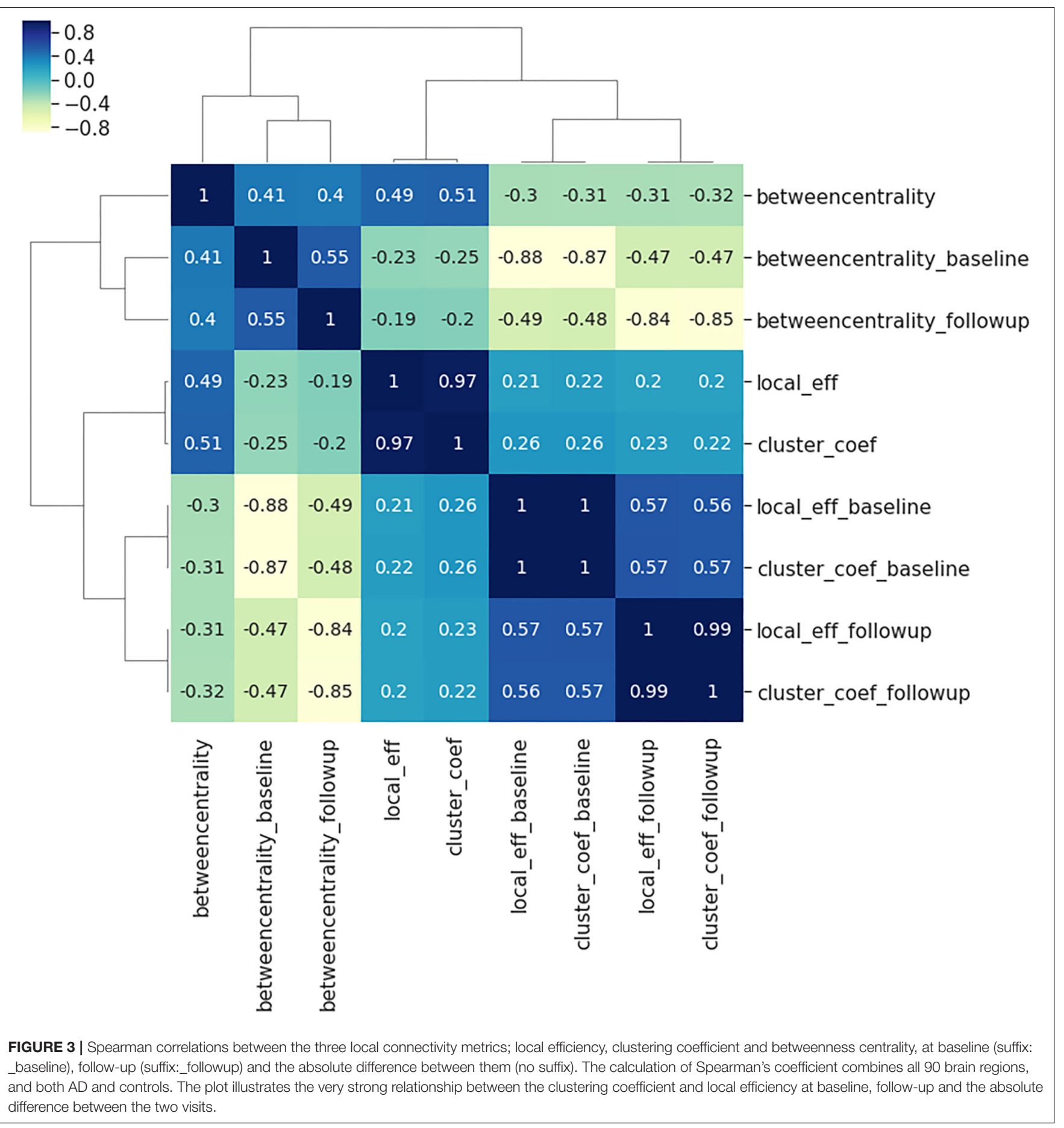

strong correlation that exists between the local efficiency and clustering coefficient at the baseline, follow-up and also, the absolute change (see Figure 3). On the other hand, Table 3 reports the top results of the association between gene expression and the change in brain global connectivity. In this case, all observed associations were not statistically significant after correcting for multiple hypotheses (threshold is stated in Table 3).

\section{Regression Analysis: The Change in Local and Global Brain Connectivity With Gene Expression}

We analyzed the directed association through regressing the change in local connectivity (as a dependant variable), at each $\mathrm{AAL}$ region, on gene expression using (as an independent variable or predictor) a quantile regression model. Table 4 
TABLE 3 | Top 20 spearman association results of the change in global network metrics with targeted AD gene expressions.

\begin{tabular}{lccl}
\hline Gene & \multicolumn{3}{c}{ Threshold $=\frac{\mathbf{0 . 0 5}}{\mathbf{1 7 \times 4}}=\mathbf{7 . 3 5 e}-\mathbf{0 4}$} \\
\cline { 2 - 4 } & $\boldsymbol{\rho}$ & $\boldsymbol{P}$-value & Global feature \\
\hline PAXIP1 & 0.3889 & 0.0069 & Transitivity \\
PLAU & -0.3824 & 0.008 & global_eff \\
ACE & -0.3696 & 0.0106 & Transitivity \\
PLAU & -0.3523 & 0.0151 & char_path_len \\
ABCA7 & -0.3492 & 0.0161 & char_path_len \\
PSEN1 & -0.299 & 0.0412 & Transitivity \\
APP & -0.2602 & 0.0774 & char_path_len \\
PLAU & -0.2542 & 0.0847 & Louvain \\
ApoE & -0.2506 & 0.0893 & char_path_len \\
ADAM10 & -0.2365 & 0.1095 & Louvain \\
ACE & 0.2291 & 0.1213 & Louvain \\
NOS3 & -0.2207 & 0.1359 & char_path_len \\
NOS3 & -0.2202 & 0.1369 & global_eff \\
ABCA7 & -0.2164 & 0.1441 & global_eff \\
HFE & -0.2012 & 0.1752 & char_path_len \\
\hline
\end{tabular}

reports the top results, along with the regression coefficient, $p$ values and $t$-test statistic. PLAU was the only significant gene, affecting the absolute change in betweenness centrality at left Fusiform gyrus (Fusiform_L) with an increase of 487.13 at each unit increase in PLAU expression ( $p=3 e-06)$. This was followed by the expression of HFE with an effect size of 0.1277 on the change in local efficiency at the right anterior cingulate and paracingulate gyri (Cingulum_Ant_R). Those observed associations are illustrated in Figure 4, and, the protein-protein interaction (Szklarczyk et al., 2016) of the aforementioned genes are shown in Figure 5. More specifically, the protein to protein interaction is the results of the query to the STRING database by using the multiple protein tools for homo sapiens, by querying for direct and 1 additional indirect interactions. Those interactions are the results of several results from past literature.

Supplementary Figures S7-S9 show the Manhattan plots for the $-\log 10$ of the $p$-values corresponding to the quantile regression models of the change in local efficiency, clustering coefficient and betweenness centrality, respectively.

Similarly, we regressed the absolute change of global connectivity measures on gene expression values and the top results are shown in Table 5.

\section{Additive Genetic Effect on Brain Regions}

To visualize the overall contribution of $\mathrm{AD}$ gene risk factors used in this work on distinct brain areas, we added up the $-\log 10 p$ values for the gene expression coefficients at each of the $90 \mathrm{AAL}$ regions. The $p$-values were obtained from the quantile regression analysis between the gene expression values and each of the three connectivity metrics-those are the absolute difference between baseline and follow-up of local efficiency, clustering coefficient and betweenness. Figure 6 summarizes this by 1) representing the brain connectome without edges for each of the connectivity metrics, 2) each node represents a distinct AAL region and is annotated with the name of the region, 3) the size of each node is the sum - $\log 10$ of the regression coefficient associated $p$-values for all the genes. It is clear from Figure 6 that local efficiency and clustering coefficient show more similar patterns of association with genes compared to betweenness centrality. This means that the gene expression has stronger association with local structure of the brain when using clustering measures (e.g., clustering coefficients), while the pattern of association tends to be weaker when using measures expressing the state of a region being between two others (e.g., betweenness centrality).

The colors are assigned automatically by the BrainNet Viewer.

Overall, although the gene contributions to the absolute change in local efficiency have a similar pattern to that of clustering coefficient, the contribution to betweenness centrality change is relatively small.

\section{Regression Analysis: The Difference in CDR With the Difference in Global and Local Connectivity}

To asses the directed and undirected association of the longitudinal measures of global connectivity and CDR scores, we calculated the difference between baseline and follow-up visits for both $\mathrm{CDR}$ and global connectivity metrics, i.e., $C D R_{\text {baseline }}-C D R_{\text {follow-up }}$ and metric $_{\text {baseline }}-$ metric $_{\text {follow-up, }}$, respectively. The Spearman and quantile regression results are both shown in Table 6. We observed a correlation between the increase of the transitivity score (global brain segregation) and the CDR memory score over time $(\beta=-6.14 e-06, p=0.0034)$. On the other hand, there is a positive correlation between global efficiency (global brain integration) and the CDR "home and hobbies" score.

Similarly, in Supplementary Table S2 we looked at the monotonic effect of local connectivity metrics on the seven CDR scores, both represented as the subtraction of the follow-up visit from the baseline visit. The increase in betweenness centrality was shown to have different effects on the CDR score over the 1-year time period. For example, as the betweenness centrality decreases over time, the judgement and problem solving increases in severity by $1.06 \mathrm{e}-08$ over time $(p=1.32 \mathrm{e}-17)$, in the frontal lobe (Frontal_Inf_Oper_L).

\section{Multivariate Analysis: Ridge Regression}

We regressed the difference in CDR visits (response variable; $\mathrm{Y}$ ), one score at a time, on both the difference in global brain connectivity (predictor; X1), one connectivity metric at a time and all gene expression values (predictor; X2), using the ridge regression model. Supplementary Table S3 reports the mean squared error (the score column) and shows the top hits in the multiple ridge regression. It shows that the $\alpha$ (alpha column) could not converge, using the cross-validation, when the response variables were judgment or personal care. However, the CDR score results show that genes and connectivity metrics have a small effect $(\beta)$ on the response variables (the change in CDR scores over time), and the larger effects were 
TABLE 4 | Top 50 quantile regression results of the change in local network metrics $(y)$ and targeted AD gene expression $(x)$.

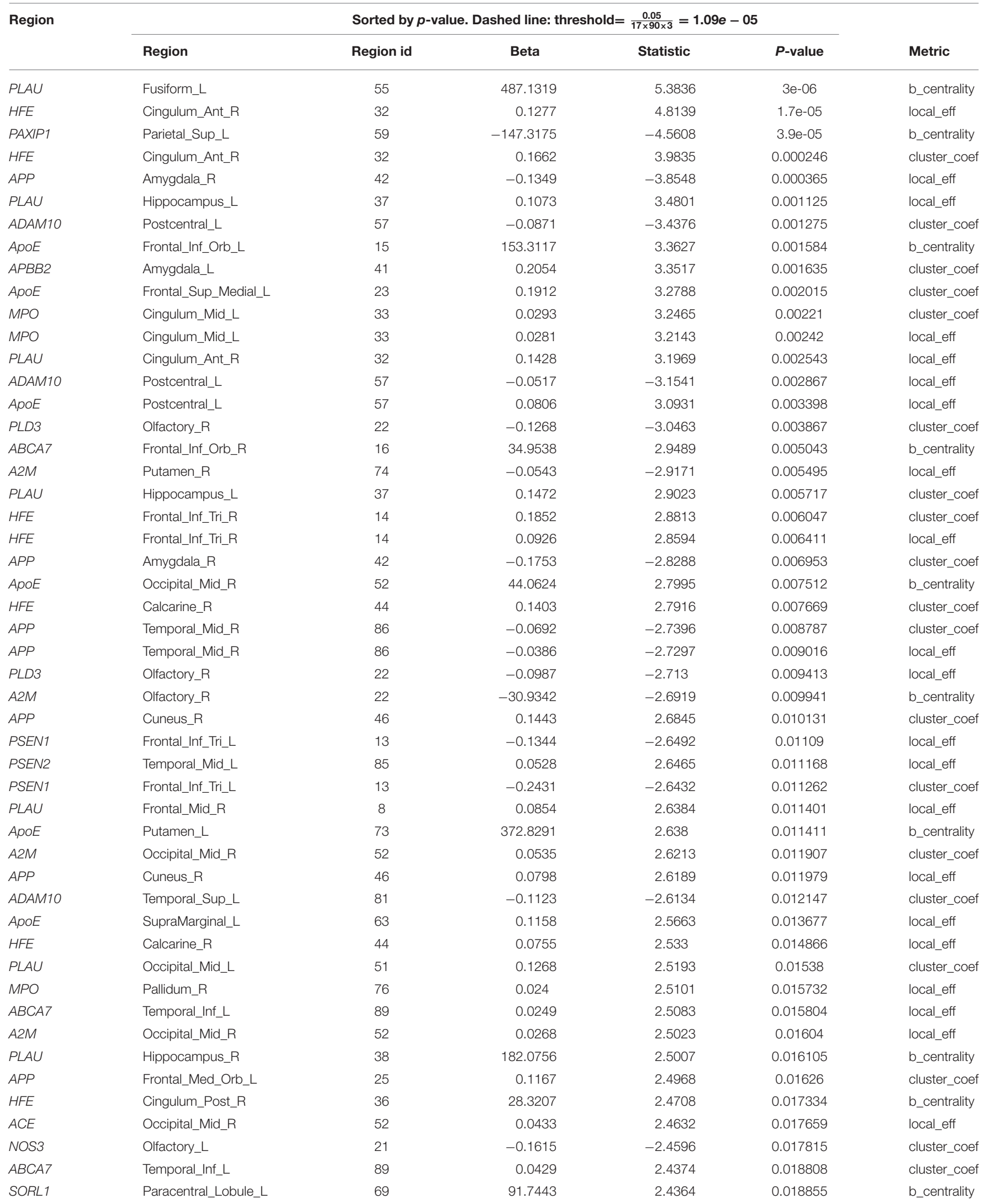



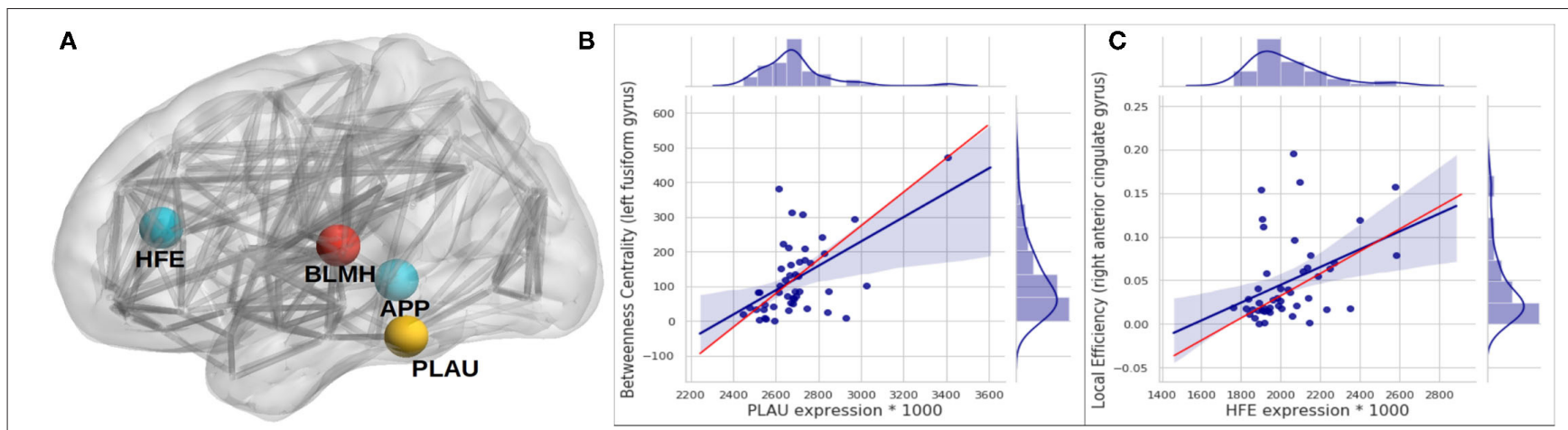

FIGURE 4 | Subfigure (A) higlights regions in the brain where significant associations-between gene expression and longitudinal change in local connectivity metrics were found, using quantile regression (HFE and PLAU) and Spearman associations (APP and BLMH). Each gene is plotted at the AAL brain region where the association was significant; APP at Temporal_Mid_R, BLMH at Heschl_L, PLAU at Fusiform_L and HFE at Cingulum_Ant_R. (B,C) are scatter plots to visualize the association between PLAU gene expression and betweenness centrality in the left fusiform gyrus (A), and between the expression of HFE gene with local efficiency in right anterior cingulate gyrus (B). The red line on the plots represents the median (quantile) regression line, while the blue line represents the ordinary least square line. It is important to bear in mind that after the multiple hypothesis testing only the PLAU gene association was still significant.

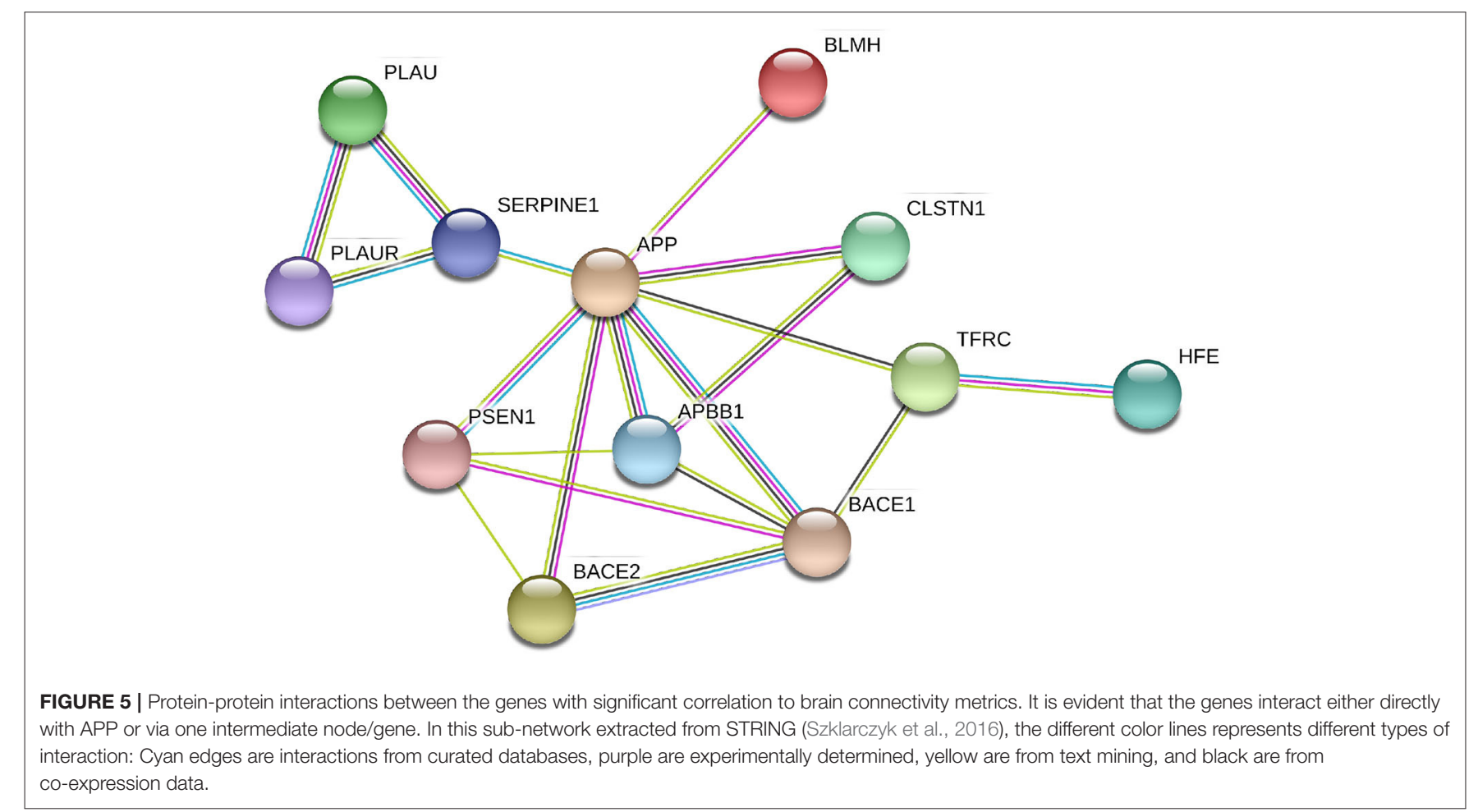

observed when using the total CDR score (CDR_diff) as a response variable. The connectivity metrics and expression of genes have both negative and positive effects on CDR change. The expression of ApoE, for example, has a negative effect $(\beta)$ of -0.24 on the change in memory score, i.e., the memory rating decreases by 0.24 as the ApoE expression increases. While when the expression of $A p o E$ increases one unit, the home and hobbies score increases, over time, by 0.12 .

\section{DISCUSSION}

In this paper we aimed to analyse the longitudinal change of local and global brain connectivity metrics in Alzheimer's Disease, using a dataset from ADNI. We evaluated the association between the most commonly used connectivity metrics and the expression of known AD risk genes. Finally, we studied the multivariate association between connectivity metrics, gene expression and dementia clinical ratings. It is worth mentioning 
TABLE 5 | Top quantile regression results of the change in global network metrics and targeted AD gene expressions.

\begin{tabular}{|c|c|c|c|c|}
\hline \multirow[t]{2}{*}{ Gene } & \multicolumn{4}{|c|}{ Threshold $\frac{0.05}{17 \times 4}=7.35 e-04$} \\
\hline & Beta & Statistic & $P$-value & Metric \\
\hline PAXIP1 & 0.0155 & 2.1179 & 0.039738 & Transitivity \\
\hline PSEN1 & -0.0366 & -2.0821 & 0.04305 & Transitivity \\
\hline$A 2 M$ & 0.0178 & 1.9728 & 0.054679 & Louvain \\
\hline$P L A U$ & -0.0157 & -1.9288 & 0.06008 & global_eff \\
\hline APBB2 & 0.0166 & 1.7579 & 0.085573 & Louvain \\
\hline$A B C A 7$ & 0.0077 & 1.5185 & 0.135881 & Transitivity \\
\hline$B L M H$ & 0.0101 & 1.3476 & 0.184529 & Transitivity \\
\hline$A C E$ & -0.0162 & -1.2618 & 0.213524 & Transitivity \\
\hline ADAM10 & -0.0147 & -1.2538 & 0.21638 & Louvain \\
\hline$A p o E$ & -0.0555 & -1.1673 & 0.249246 & char_path_len \\
\hline PLD3 & -0.0096 & -1.1433 & 0.258978 & Transitivity \\
\hline$A B C A 7$ & -0.0156 & -1.1367 & 0.261684 & char_path_len \\
\hline SORL 1 & 0.0147 & 1.1278 & 0.265372 & Transitivity \\
\hline$A p o E$ & -0.0099 & -1.1048 & 0.275101 & global_eff \\
\hline HFE & -0.0161 & -1.0436 & 0.302239 & Louvain \\
\hline
\end{tabular}

Threshold $=\frac{0.05}{17 \times 4}=7.35 e-04$.

that it is not practical to do expression analysis on brain tissues, thus, we had to use a proxy for what is generally happening in the body by using the blood samples. This is the closest proxy that is feasible when working with both patients and controls, also in the view of using the proposed biomarkers in daily practice. Moreover, we verified that the found genes were expressed in the brain parenchyma by using the ISH Allen Atlas (Sunkin et al., 2012) and the Protein Atlas (Sjöstedt et al., 2020), which confirmed positively their expression.

Our results show that Alzheimer's risk genes can manipulate the amount of change observed in the structural connectome, measured as the absolute difference of longitudinal connectivity metrics. We also show that longitudinal regional connectivity metrics, global brain segregation and integration have effects on the CDR scores. More specifically, as the disease progresses, we observe a correlation between brain segregation and cognitive decline, the latter is measured as the memory CDR score. We also noticed a correlation between the global efficiency and the home and hobbies CDR scores (see Table 6). Furthermore, we observe a consistent decrease, over time (though did not hit the significance threshold), in the local efficiency (a network connectivity metric of a node is defined as the inverse of the shortest average path between any pair of that node's neighbors Rubinov and Sporns, 2010) at the right middle temporal gyrus (Temporal_Mid_R; see Table 2) in response to an increase in APP expression. The same connectivity metric showed another potential increase over time at the right anterior cingulate and paracingulate gyri (see Table 4) as the expression of HFE increases.

Prescott et al. (2014) have investigated the differences in the structural connectome at the three clinical stages of $\mathrm{AD}$ using a cross-sectional study design. They targeted regional brain areas that are known to have increased amyloid plaque. Their work suggested that connectome damage might occur at an earlier preclinical stage toward developing AD. Here, we further adapted a longitudinal study design and incorporated known $\mathrm{AD}$ risk genes in analyzing the changes in the connectome. We specifically focus on how the damage in the connectome is associated with gene expression and how is that change in connectome affects dementia symptoms, globally and locally at distinct brain regions. Aside from our previous work which examined the $A p o E$ associations with longitudinal global connectivity in $\mathrm{AD}$ using longitudinal global connectivity metrics (Elsheikh et al., 2020b), this study, to our knowledge, is the first of its type to include gene expression data in a longitudinal analysis of global and local brain connectivity. However, similar work has been done in schizophrenia where the association between longitudinal magnetic resonance imaging features, derived from the DWI, and higher genetic risk for schizophrenia were investigated using structural brain connectivity (Alloza et al., 2018).

The expression in the regional areas of the brain where we found significant (i.e., PLAU) or potential (i.e., HFE, APP, and $\mathrm{BLMH}$ ) association with local connectivity are highlighted in Figure 4. Those are mostly in line with previous findings of the regional molecular properties in the brain (e.g., Grothe et al., 2018). In this study we found that the Plasminogen activator, urokinase (PLAU) expression affects the betweenness centrality (a measure of the node's contribution to the flow of information in a network Rubinov and Sporns, 2010) in the left fusiform gyrus, over time (see Figure 4 and Table 4). Although the functionality of this region is not fully understood, its relationship with cognition and semantic memory was previously reported (Galton et al., 2001). PLAU, on the other hand, was shown to be a risk factor in the development of late-onset $\mathrm{AD}$ as a result of its involvement in the conversion of plasminogen to plasmin-a contributor to the processing of APP by the urokinase-type plasminogen activator (Finckh et al., 2003).

Among further results-including those not surviving the multiple hypothesis testing-potentially align with findings in the literature of genetics and neuroimaging. Robson et al. (2004) studied the interaction of the $C 282 Y$ allele $H F E$ - the common basis of hemochromatosis-and found that carriers of ApoE-4, the $\mathrm{C} 2$ variant in $\mathrm{TF}$ and $\mathrm{C} 282 \mathrm{Y}$ are at higher risk of developing $\mathrm{AD}$. The HFE gene is also known for regulating iron absorption, which results in recessive genetic disorders, such as hereditary haemochromatosis also related to AD (Pilling et al., 2019). These studies align with our findings where we show that HFE expression (though it did not service the significance threshold after multiple testing correction) can potentially affect the local efficiency at the right anterior cingulate gyrus (see Figure 4 and Table 4), which might indicate a possible effect of HFE expression on the personality of AD patients or those at risk of developing the disease. When examining the linear associations between gene expression and local connectivity (see Table 2 and Supplementary Figure S6), we found that the local efficiency in the right middle temporal gyrus (nearly hit the significance threshold), known for its involvement in cognitive processes including comprehension of language, negatively associates with $A P P$ expression. We also found a potential correlation between 


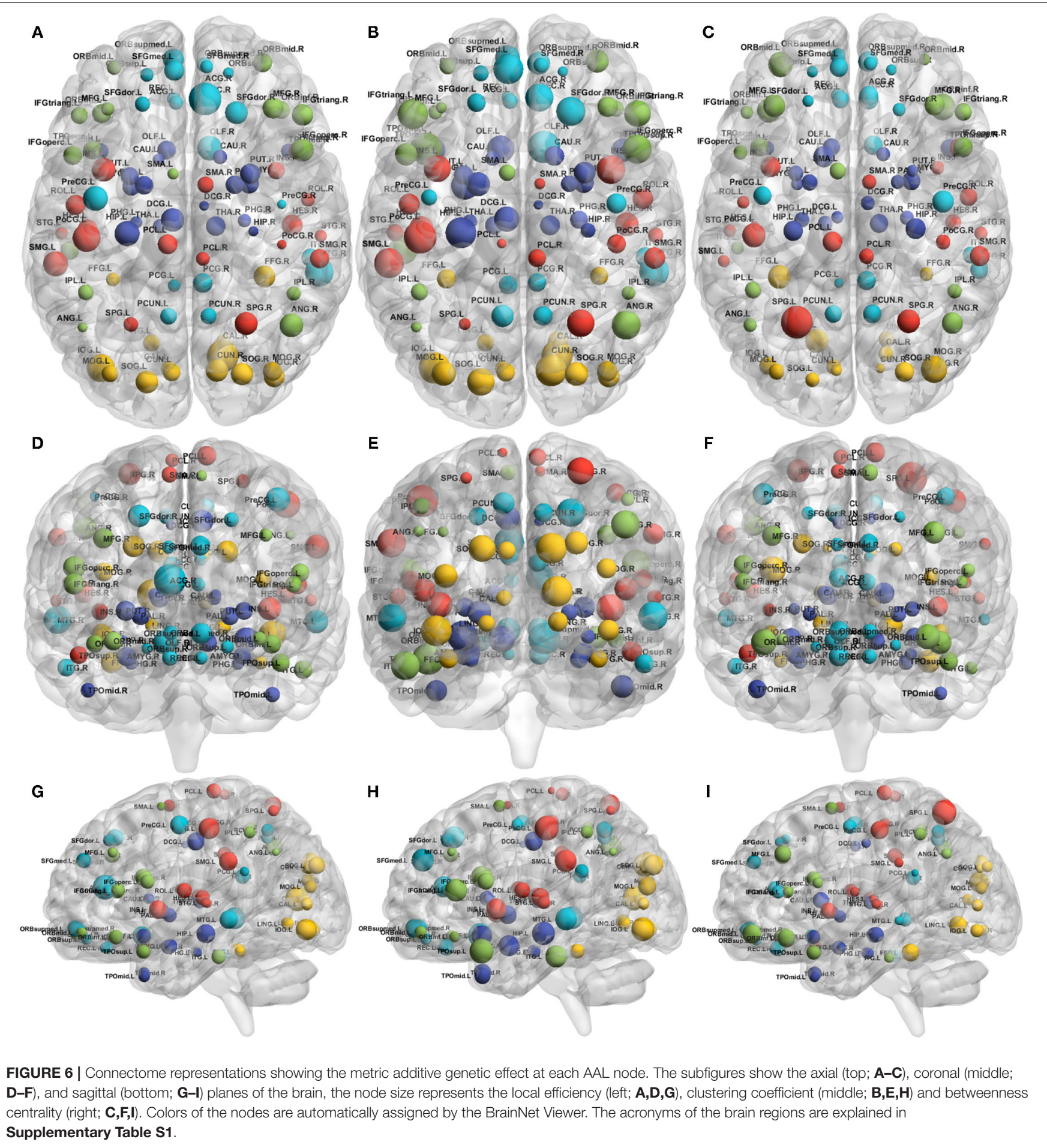

the left Heschl gyrus's clustering coefficient and the bleomycin hydrolase (BLMH) expression. In the human brain, the BLMH protein is expressed in the neocortical neurons and associated to misfolded proteins related to AD. Some studies (Farrer et al., 1998; Papassotiropoulos et al., 2000) have found that a variant in the $B L M H$ gene, which leads to the Ile $443 \rightarrow$ Val in the BLMH protein, increases the risk of $\mathrm{AD}$; this was strongly marked in $A p o E-4$ carriers. The BLMH protein can process the $A \beta$ protein precursor, and is involved in the production of $A \beta$ peptide (Kajiya et al., 2006).

The regional expression of the APP gene has been shown to be positively correlated with the severity of regional amyloid 
TABLE 6 | Quantile regression results of the difference in CDR $(y)$ with the difference in global connectivity $(x)$.

\begin{tabular}{|c|c|c|c|c|c|c|}
\hline Global metric & \multicolumn{6}{|c|}{ Threshold $=\frac{0.05}{6 \times 4}=2.08 \mathrm{e}-3 .{ }^{*}$ represents significant $p$-value } \\
\hline Transitivity & $-6.14 \mathrm{e}-06\left(0.0034^{*}\right)$ & $-1.8 \mathrm{e}-06$ (nan) & $-3.2 \mathrm{e}-07$ (nan) & $8.4 \mathrm{e}-07$ (0.9249) & $4.13 e-06(0.7324)$ & $-3.1 \mathrm{e}-07$ (nan) \\
\hline global_eff & $1.3 e-06(0.8572)$ & $3.36 \mathrm{e}-06(0.9944)$ & $3.5 e-07$ (0.9826) & $3.09 \mathrm{e}-06(0.3131)$ & $9.5 \mathrm{e}-06(0.1613)$ & $-2.5 \mathrm{e}-07$ (nan) \\
\hline Louvain & $-2.64 \mathrm{e}-06(0.1683)$ & $-6.84 \mathrm{e}-06(0.0352)$ & $1.21 \mathrm{e}-06(0.0012)$ & $-1.01 \mathrm{e}-06(0.8125)$ & $-2.16 e-06(0.9424)$ & $-6.3 e-07(0.1787)$ \\
\hline Spearman: & $\rho$ (p-value) & & & & & \\
\hline transitivity & $-0.3395(0.021)$ & $-0.0763(0.6142)$ & $-0.0618(0.6835)$ & 0.0661 (0.6623) & $0.161(0.2851)$ & $-0.0081(0.9574)$ \\
\hline global_eff & $0.0483(0.7497)$ & $0.0056(0.9707)$ & 0.0505 (0.7388) & $0.2685(0.0712)$ & $0.4145\left(0.0042^{*}\right)$ & $-0.0263(0.8625)$ \\
\hline Louvain & $-0.1955(0.1928)$ & $-0.3119(0.0349)$ & $0.2077(0.166)$ & $-0.0968(0.522)$ & $-0.11(0.4666)$ & $-0.0628(0.6784)$ \\
\hline char_path_len & $-0.1183(0.4337)$ & $-0.0516(0.7333)$ & $-0.0281(0.8531)$ & $-0.2909(0.0498)$ & $-0.3811(0.009)$ & $-0.0065(0.9658)$ \\
\hline
\end{tabular}

deposition observed in PET studies. The temporal medial region and the fusiform gyrus of the brain are two of the most affected regions by amyloid deposition and have high levels of APP expression (Grothe et al., 2018). Recent findings from tau-sensitive positron emission tomography data have also confirmed the spatial correspondence between accumulation of tau pathology and neurodegeneration in $\mathrm{AD}$ patients, within the same regions, though only correlations with the ApoE genes were investigated (Cho et al., 2016). The BLMH protein alters the processing of APP and significantly increases the release of its proteolytic fragment. It has been previously reported to be expressed and have an impact on the hippocampal tissues, but not investigated in other brain regions (Suszyńska-Zajczyk et al., 2014). To our knowledge, apart from the general expression in the brain parenchyma reported in the Allen brain atlas (Hawrylycz et al., 2012) and Protein Atlas Sjöstedt et al. (2020), no study has shown spatial expression of the PLAU and HFE genes among AD patients. Nevertheless, we hypothesize that there is a potential correlation of these gene expression in specific nodes of the brain connectome, and that it is related to their interaction with the APP gene which is particularly expressed in the nodes highlighted in Figure 4. This hypothesis is also supported by the protein-protein interaction shown in Figure 5.

Even though none of the AD risk genes showed a significant effect on the longitudinal change in global connectivity (see Tables 3, 5), some of these genes showed significant effects on local connectivity changes at specific brain areas (see Tables 2, 4). The global connectivity metrics of the brain, on the other hand, have shown promising results in affecting the change observed in CDR scores, including memory, judgement and problem solving, as well as home and hobbies, as shown in Table 6. Previous work studied the association between genome-wide variants and global connectivity in $\mathrm{AD}$, represented as brain integration and segregation, and found that some genes affect the amount of change observed in global connectivity measures (Elsheikh et al., $2020 \mathrm{~b}$ ). This suggests that a generalization of the current study at a gene-wide level might warrant further analysis.
Considering the possible redundancy of brain connectivity metrics (Rubinov, 2016) studied here, we looked for correlations with the nodal degrees and other features, and observed a relative correlation with betweenness centrality (see Figure 2). When we compared all the metrics to each other, we only found a correlation between local efficiency and cluster coefficient metrics. Therefore, we hypothesize that the degree is a simpler representation than betweenness centrality that could be used as a substitute. However, this was not the case with other metrics. We suggest either clustering coefficient or local efficiency should also be investigated in similar studies. More generally, when correcting our threshold, we did not consider the number of metrics, because of the high co-linearity. Therefore, we believe that reporting all, or only one, did not affect the results.

Our work provides new insights into the progression of Alzheimer's Disease, though replication on a larger sample size is required. Indeed, one limitation here was the small sample size available, as we needed to narrow down our selection of participants to those who 1) attended both the baseline and follow-up visits, 2) have their CDR scores measured, 3) have their genetic and imaging information available. Another limitation is the use of only two time points, the baseline and the first followup visits. Other datasets do not offer all those data, and indeed even recent studies have been published with similar sample sizes (Kim et al., 2020). Moreover, the limited availability of samples does not allow capturing the effects of connectivity changes over a longer-term or studying the survival probabilities in AD. Extending to more time points would have been useful, but it would have reduced the dataset further. We foresee future work in using a more complex unified multi-scale model to facilitate studying the multivariate effect of clinical and genetic factors on brain diseases, besides considering the complex interplay of genetic factors (Elsheikh et al., 2020a). Moreover, previous studies with similar sample sizes have been able to provide relevant insights for the gene and brain interaction networks showing the validity of the approach (Richiardi et al., 2015). 
This analysis was conducted for $\mathrm{AD}$, using a longitudinal study design, and highlighted the role of PLAU, HFE, APP and $B L M H$ in affecting the pattern information propagated in particular regions of the brain, which might have a direct effect on a person's recognition and cognitive abilities. The four genes were previously shown to be expressed in both the temporal and visual cortex in $\mathrm{AD}$, according to the Allen Human Brain Atlas https:/human.brain-map.org/ish/ search and in the whole brain with low regional specificity according to the Protein Atlas https://www.proteinatlas.org. Furthermore, the results illustrated the effect of brain structural connections on memory and cognitive process, especially the capacity for reaching a decision or drawing conclusions. The findings presented here might provide better in-vivo estimation of local neurodegneration and related treatments. The Braak staging hypothesis is still controversial, and other in-vivo studies have shown regional effects using positron emission tomography (PET) imaging (temporal lobe, the anterior cingulate gyrus, and the parietal operculum) (Grothe et al., 2018). These studies have also shown an impact on the default mode network connectivity (Palmqvist et al., 2017). Therefore, further investigation of regional patterns is relevant. Very recent results on treatments in mice showed that drug based modulated neuronal activity can reduce amyloid plaques in specific locations and circuitry (Canter et al., 2019). In view of future treatments based on specific spatial location and genetic influences, our study provides some initial insights into connectivity outcomes, and to some extent, enhances our understanding of the regions/circuits that show amyloid aggregation or neurodegeneration.

\section{DATA AVAILABILITY STATEMENT}

The original contributions presented in the study are included in the article/Supplementary Material, further inquiries can be directed to the corresponding authors.

\section{ETHICS STATEMENT}

Data were acquired in previous studies within the Alzheimer's Disease Neuroimaging initiative which was approved by local committee https://adni.loni.usc.edu/. The patients/participants provided their written informed consent to participate in this study.

\section{AUTHOR CONTRIBUTIONS}

SE conducted the integrated imaging-genetics analysis and wrote the manuscript. AC preprocessed all neuroimaging data and set the connectome pipeline. NM and AC gave constructive feedback on this work continuously and followed up the analysis and writing progress closely. EC gave feedback on the overall manuscript. The final version of the paper was proofread by all authors.

\section{FUNDING}

We would like to acknowledge our funders, the Organisation for Women in Science for the Developing World (OWSD), the Swedish International Development Cooperation Agency (SIDA) and the University of Cape Town for their continuous support. Partly supported by the European Union's Horizon 2020 research and innovation programme under grant agreement Sano no 857533, and by the International Research Agendas programme of the Foundation for Polish Science, cofinanced by the European Union under the European Regional Development Fund.

\section{ALZHEIMER'S DISEASE NEUROIMAGING INITIATIVE}

Data collection and sharing for this project was funded by the Alzheimer's Disease Neuroimaging Initiative (ADNI) (National Institutes of Health Grant U01 AG024904) and DOD ADNI (Department of Defense award number W81XWH-12-2-0012). ADNI is funded by the National Institute on Aging, the National Institute of Biomedical Imaging and Bioengineering, and through generous contributions from the following: AbbVie, Alzheimer's Association; Alzheimer's Drug Discovery Foundation; Araclon Biotech; BioClinica, Inc.; Biogen; BristolMyers Squibb Company; CereSpir, Inc.; Cogstate; Eisai Inc.; Elan Pharmaceuticals, Inc.; Eli Lilly and Company; EuroImmun; F. Hoffmann-La Roche Ltd and its affiliated company Genentech, Inc.; Fujirebio; GE Healthcare; IXICO Ltd.; Janssen Alzheimer's Immunotherapy Research \& Development, LLC.; Johnson \& Johnson Pharmaceutical Research \& Development LLC.; Lumosity; Lundbeck; Merck \& Co., Inc.; Meso Scale Diagnostics, LLC.; NeuroRx Research; Neurotrack Technologies; Novartis Pharmaceuticals Corporation; Pfizer Inc.; Piramal Imaging; Servier; Takeda Pharmaceutical Company; and Transition Therapeutics. The Canadian Institutes of Health Research is providing funds to support ADNI clinical sites in Canada. Private sector contributions are facilitated by the Foundation for the National Institutes of Health (www.fnih.org). The grantee organization is the Northern California Institute for Research and Education, and the study is coordinated by the Alzheimer's Therapeutic Research Institute at the University of Southern California. ADNI data are disseminated by the Laboratory for Neuro Imaging at the University of Southern California.

\section{SUPPLEMENTARY MATERIAL}

The Supplementary Material for this article can be found online at: https://www.frontiersin.org/articles/10.3389/fnhum. 2021.761424/full\#supplementary-material 


\section{REFERENCES}

Aganj, I., Lenglet, C., Sapiro, G., Yacoub, E., Ugurbil, K., and Harel, N. (2010). Reconstruction of the orientation distribution function in single-and multipleshell q-ball imaging within constant solid angle. Magn. Reson. Med. 64, 554-566. doi: 10.1002/mrm.22365

Alexander, A. L., Lee, J. E., Lazar, M., and Field, A. S. (2007). Diffusion tensor imaging of the brain. Neurotherapeutics 4, 316-329. doi: $10.1016 /$ j.nurt.2007.05.011

Alloza, C., Cox, S. R., Cábez, M. B., Redmond, P., Whalley, H., Ritchie, S., et al. (2018). Polygenic risk score for schizophrenia and structural brain connectivity in older age: a longitudinal connectome and tractography study. Neuroimage 183, 884-896. doi: 10.1016/j.neuroimage.2018.08.075

Barnes, D. E., and Yaffe, K. (2011). The projected effect of risk factor reduction on Alzheimer's disease prevalence. Lancet Neurol. 10, 819-828. doi: 10.1016/S1474-4422(11)70072-2

Brown, J., Terashima, K., Burggren, A., Ercoli, L., Miller, K., and other (2011). Brain network local interconnectivity loss in aging apoe-4 allele carriers. Proc. Natl. Acad. Sci. U.S.A. 108, 20760-20765. doi: 10.1073/pnas.1109038108

Canter, R. G., Huang, W.-C., Choi, H., Wang, J., Watson, L. A., Yao, C. G., et al. (2019). 3d mapping reveals network-specific amyloid progression and subcortical susceptibility in mice. Commun. Biol. 2, 1-12. doi: $10.1038 /$ s42003-019-0599-8

Chen, Y., Chen, K., Zhang, J., Li, X., Shu, N., Wang, J., et al. (2015). Disrupted functional and structural networks in cognitively normal elderly subjects with the APOE $\epsilon 4$ allele. Neuropsychopharmacology 40:1181. doi: 10.1038/npp.2014.302

Chiesa, P. A., Cavedo, E., Lista, S., Thompson, P. M., Hampel, H., Initiative, A. P. M., et al. (2017). Revolution of resting-state functional neuroimaging genetics in Alzheimer's disease. Trends Neurosci. 40, 469-480. doi: 10.1016/j.tins.2017.06.002

Cho, H., Choi, J. Y., Hwang, M. S., Lee, J. H., Kim, Y. J., Lee, H. M., et al. (2016). Tau PET in Alzheimer disease and mild cognitive impairment. Neurology 87, 375-383. doi: 10.1212/WNL.0000000000002892

Cohen, J. R., and D'Esposito, M. (2016). The segregation and integration of distinct brain networks and their relationship to cognition. J. Neurosci. 36, 12083-12094. doi: 10.1523/JNEUROSCI.2965-15.2016

Corder, E., Saunders, A., Strittmatter, W. J., Schmechel, D. E., Gaskell, P. C., Small, G. W., et al. (1993). Gene dose of apolipoprotein E type 4 allele and the risk of Alzheimer's disease in late onset families. Science 261, 921-923. doi: $10.1126 /$ science. 8346443

Deco, G., Tononi, G., and Boly, M. (2015). Rethinking segregation and integration: contributions of whole-brain modelling. Nat. Rev. Neurosci. 16, 430-439. doi: $10.1038 / \mathrm{nrn} 3963$

Douaud, G., Smith, S., Jenkinson, M., Behrens, T., Johansen-Berg, H., Vickers, J., et al. (2007). Anatomically related grey and white matter abnormalities in adolescent-onset schizophrenia. Brain 130, 2375-2386. doi: 10.1093/brain/awm184

Elsheikh, S. S., Chimusa, E. R., Crimi, A., and Mulder, N. J. (2020a). Bigen: integrative clinical and brain-imaging genetics analysis using structural equation model. bioRxiv. doi: 10.1101/2020.02.04.934596

Elsheikh, S. S., Chimusa, E. R., Mulder, N. J., and Crimi, A. (2020b). Genome-wide association study of brain connectivity changes for Alzheimer's disease. Sci. Rep. 10, 1-16. doi: 10.1038/s41598-020-58291-1

Escott-Price, V., Bellenguez, C., Wang, L.-S., Choi, S.-H., Harold, D., Jones, L., et al. (2014). Gene-wide analysis detects two new susceptibility genes for Alzheimer's disease. PLoS ONE 9:e94661. doi: 10.1371/journal.pone.0094661

Farrer, L. A., Abraham, C. R., Haines, J. L., Rogaeva, E. A., Song, Y., McGraw, W. T., et al. (1998). Association between bleomycin hydrolase and Alzheimer's disease in caucasians. Ann. Neurol. 44, 808-811. doi: 10.1002/ana.410440515

Finckh, U., Van Hadeln, K., Müller-Thomsen, T., Alberici, A., Binetti, G., Hock, C., et al. (2003). Association of late-onset Alzheimer disease with a genotype of plau, the gene encoding urokinase-type plasminogen activator on chromosome 10q22. 2. Neurogenetics 4, 213-217. doi: 10.1007/s10048-003-0157-9

Gaiteri, C., Mostafavi, S., Honey, C. J., De Jager, P. L., and Bennett, D. A. (2016). Genetic variants in Alzheimer disease-molecular and brain network approaches. Nat. Rev. Neurol. 12, 413. doi: 10.1038/nrneurol.2016.84
Galton, C. J., Patterson, K., Graham, K., Lambon-Ralph, M. A., Williams, G., Antoun, N., et al. (2001). Differing patterns of temporal atrophy in Alzheimer's disease and semantic dementia. Neurology 57, 216-225. doi: 10.1212/WNL.57.2.216

Garyfallidis, E., Brett, M., Amirbekian, B., Rokem, A., Van Der Walt, S., Descoteaux, M., et al. (2014). Dipy, a library for the analysis of diffusion MRI data. Front. Neuroinform. 8:8. doi: 10.3389/fninf.2014.00008

Gatz, M., Pedersen, N. L., Berg, S., Johansson, B., Johansson, K., Mortimer, J. A., et al. (1997). Heritability for Alzheimer's disease: the study of dementia in Swedish twins. J. Gerontol. A Biol. Sci. Med. Sci. 52, M117-M125. doi: 10.1093/gerona/52A.2.M117

Goate, A., Chartier-Harlin, M.-C., Mullan, M., Brown, J., Crawford, F., Fidani, L., et al. (1991). Segregation of a missense mutation in the amyloid precursor protein gene with familial Alzheimer's disease. Nature 349, 704. doi: 10.1038/ $349704 \mathrm{a} 0$

Gordon, E. M., Laumann, T. O., Adeyemo, B., Huckins, J. F., Kelley, W. M., and Petersen, S. E. (2014). Generation and evaluation of a cortical area parcellation from resting-state correlations. Cereb. Cortex 26, 288-303. doi: 10.1093/cercor/bhu239

Griffa, A., Baumann, P. S., Thiran, J.-P., and Hagmann, P. (2013). Structural connectomics in brain diseases. Neuroimage 80, 515-526. doi: 10.1016/j.neuroimage.2013.04.056

Grothe, M. J., Sepulcre, J., Gonzalez-Escamilla, G., Jelistratova, I., Schöll, M., Hansson, O., et al. (2018). Molecular properties underlying regional vulnerability to Alzheimer's disease pathology. Brain 141, 2755-2771. doi: 10.1093/brain/awy189

Hagmann, P., Cammoun, L., Gigandet, X., Meuli, R., Honey, C. J., Wedeen, V. J., et al. (2008). Mapping the structural core of human cerebral cortex. PLoS Biol. 6:e159. doi: 10.1371/journal.pbio.0060159

Hawrylycz, M., Miller, J. A., Menon, V., Feng, D., Dolbeare, T., GuillozetBongaarts, A. L., et al. (2015). Canonical genetic signatures of the adult human brain. Nat. Neurosci. 18, 1832-1844. doi: 10.1038/nn.4171

Hawrylycz, M. J., Lein, E. S., Guillozet-Bongaarts, A. L., Shen, E. H., Ng, L., Miller, J. A., et al. (2012). An anatomically comprehensive atlas of the adult human brain transcriptome. Nature 489, 391. doi: 10.1038/nature11405

Hoerl, A. E., and Kennard, R. W. (1970). Ridge regression: biased estimation for nonorthogonal problems. Technometrics 12, 55-67. doi: 10.1080/00401706.1970.10488634

Irizarry, R., et al. (2003). Exploration, normalization, and summaries of high density oligonucleotide array probe level data. Biostatistics 4, 249-264. doi: 10.1093/biostatistics/4.2.249

Jahanshad, N., Rajagopalan, P., Hua, X., Hibar, D., Nir, T. M., Toga, A. W., et al. (2013). Genome-wide scan of healthy human connectome discovers SPON1 gene variant influencing dementia severity. Proc. Natl. Acad. Sci. U.S.A. 110, 4768-4773. doi: 10.1073/pnas.1216206110

Kajiya, A., Kaji, H., Isobe, T., and Takeda, A. (2006). Processing of amyloid $\beta$ peptides by neutral cysteine protease bleomycin hydrolase. Protein Pept. Lett. 13, 119-123. doi: 10.2174/092986606775101562

Kim, H.-R., Lee, T., Choi, J. K., Jeong, Y., and ADNI (2020). Genetic variants beyond amyloid and tau associated with cognitive decline. Neurology 95, e2366-e2377. doi: 10.1212/WNL.0000000000010724

Koenker, R., and Hallock, K. F. (2001). Quantile regression. J. Econ. Perspect. 15, 143-156. doi: 10.1257/jep.15.4.143

Lambert, J.-C., Ibrahim-Verbaas, C. A., Harold, D., Naj, A. C., Sims, R., Bellenguez, C., et al. (2013). Meta-analysis of 74,046 individuals identifies 11 new susceptibility loci for Alzheimer's disease. Nat. Genet. 45, 1452. doi: 10.1038/ng.2802

Levy-Lahad, E., Wasco, W., Poorkaj, P., Romano, D. M., Oshima, J., Pettingell, W. H., et al. (1995). Candidate gene for the chromosome 1 familial Alzheimer's disease locus. Science 269, 973-977. doi: 10.1126/science.7638622

Morris, J. C., Ernesto, C., Schafer, K., Coats, M., Leon, S., Sano, M., et al. (1997). Clinical dementia rating training and reliability in multicenter studies: the Alzheimer's disease cooperative study experience. Neurology 48, 1508-1510. doi: 10.1212/WNL.48.6.1508

Narum, S. R. (2006). Beyond Bonferroni: less conservative analyses for conservation genetics. Conserv. Genet. 7, 783-787. doi: 10.1007/s10592-005-9056-y 
Palmqvist, S., Schöll, M., Strandberg, O., Mattsson, N., Stomrud, E., Zetterberg, H., et al. (2017). Earliest accumulation of $\beta$-amyloid occurs within the defaultmode network and concurrently affects brain connectivity. Nat. Commun. 8, 1-13. doi: 10.1038/s41467-017-01150-x

Papassotiropoulos, A., Bagli, M., Jessen, F., Frahnert, C., Rao, M., Maier, W., et al. (2000). Confirmation of the association between bleomycin hydrolase genotype and Alzheimer's disease. Mol. Psychiatry 5, 213. doi: 10.1038/sj.mp.4000656

Pilling, L. C., Tamosauskaite, J., Jones, G., Wood, A. R., Jones, L., Kuo, C.-L., et al. (2019). Common conditions associated with hereditary haemochromatosis genetic variants: cohort study in UK biobank. bmj 364:k5222. doi: 10.1136/bmj.k5222

Prescott, J. W., Guidon, A., Doraiswamy, P. M., Roy Choudhury, K., Liu, C., Petrella, J. R., et al. (2014). The Alzheimer structural connectome: changes in cortical network topology with increased amyloid plaque burden. Radiology 273, 175-184. doi: 10.1148/radiol.14132593

Richiardi, J., Altmann, A., Milazzo, A.-C., Chang, C., Chakravarty, M. M., Banaschewski, T., et al. (2015). Correlated gene expression supports synchronous activity in brain networks. Science 348, 1241-1244. doi: 10.1126/science. 1255905

Robson, K., Lehmann, D., Wimhurst, V., Livesey, K., Combrinck, M., Merryweather-Clarke, A., et al. (2004). Synergy between the c2 allele of transferrin and the c282y allele of the haemochromatosis gene (hfe) as risk factors for developing Alzheimer's disease. J. Med. Genet. 41, 261-265. doi: 10.1136/jmg.2003.015552

Rogaev, E., Sherrington, R., Rogaeva, E., Levesque, G., Ikeda, M., Liang, Y., et al. (1995). Familial Alzheimer's disease in kindreds with missense mutations in a gene on chromosome 1 related to the Alzheimer's disease type 3 gene. Nature 376, 775. doi: 10.1038/376775a0

Rubinov, M. (2016). Constraints and spandrels of interareal connectomes. Nat. Commun. 7, 1-11. doi: 10.1038/ncomms 13812

Rubinov, M., and Sporns, O. (2010). Complex network measures of brain connectivity: uses and interpretations. Neuroimage 52, 1059-1069. doi: 10.1016/j.neuroimage.2009.10.003

Rudie, J. D., Brown, J., Beck-Pancer, D., Hernandez, L., Dennis, E., Thompson, P., et al. (2013). Altered functional and structural brain network organization in autism. Neuroimage Clin. 2, 79-94. doi: 10.1016/j.nicl.2012.11.006

Saykin, A., Shen, L., Yao, X., Kim, S., Nho, K., Risacher, S. L., et al. (2015). Genetic studies of quantitative MCI and AD phenotypes in ADNI: progress, opportunities, and plans. Alzheimers Dement. 11, 792-814. doi: 10.1016/j.jalz.2015.05.009

Schadt, E. E., Woo, S., and Hao, K. (2012). Bayesian method to predict individual snp genotypes from gene expression data. Nat. Genet. 44, 603. doi: $10.1038 / \mathrm{ng} .2248$

Sepulcre, J., Grothe, M. J., Uquillas, F., d., Ortiz-Terán, L., Diez, I., et al. (2018). Neurogenetic contributions to amyloid beta and tau spreading in the human cortex. Nat. Med. 24, 1910-1918. doi: 10.1038/s41591-018-0206-4

Shaw, P., Lerch, J. P., Pruessner, J. C., Taylor, K. N., Rose, A. B., Greenstein, D., et al. (2007). Cortical morphology in children and adolescents with different apolipoprotein e gene polymorphisms: an observational study. Lancet Neurol. 6, 494-500. doi: 10.1016/S1474-4422(07)70106-0

Sjöstedt, E., Zhong, W., Fagerberg, L., Karlsson, M., Mitsios, N., Adori, C., et al. (2020). An atlas of the protein-coding genes in the human, pig, and mouse brain. Science 367:eaay5947. doi: 10.1126/science.aay5947

Smedley, D., Haider, S., Durinck, S., Pandini, L., Provero, P., Allen, J., et al. (2015). The biomart community portal: an innovative alternative to large, centralized data repositories. Nucleic Acids Res. 43, W589-W598. doi: 10.1093/nar/gkv350

Smith, R. E., Tournier, J.-D., Calamante, F., and Connelly, A. (2012). Anatomicallyconstrained tractography: improved diffusion MRI streamlines tractography through effective use of anatomical information. Neuroimage 62, 1924-1938. doi: 10.1016/j.neuroimage.2012.06.005

Strang, G. (1993). Introduction to Linear Algebra, Vol. 3. Wellesley, MA: WellesleyCambridge Press.

Sunkin, S. M., Ng, L., Lau, C., Dolbeare, T., Gilbert, T. L., Thompson, C. L., et al. (2012). Allen brain atlas: an integrated spatio-temporal portal for exploring the central nervous system. Nucleic Acids Res. 41, D996-D1008. doi: $10.1093 / \mathrm{nar} / \mathrm{gks} 1042$

Suszyńska-Zajczyk, J., Łuczak, M., Marczak, Ł., and Jakubowski, H. (2014). Hyperhomocysteinemia and bleomycin hydrolase modulate the expression of mouse brain proteins involved in neurodegeneration. J. Alzheimers Dis. 40, 713-726. doi: 10.3233/JAD-132033

Szklarczyk, D., Morris, J. H., Cook, H., Kuhn, M., Wyder, S., Simonovic, M., et al. (2016). The string database in 2017: quality-controlled proteinprotein association networks, made broadly accessible. Nucleic Acids Res. 45, D362-D368. doi: 10.1093/nar/gkw937

Thompson, P. M., Stein, J. L., Medland, S. E., Hibar, D. P., Vasquez, A. A., Renteria, M. E., et al. (2014). The enigma consortium: large-scale collaborative analyses of neuroimaging and genetic data. Brain Imaging Behav. 8, 153-182. doi: 10.1007/s11682-013-9269-5

Tzourio-Mazoyer, N., Landeau, B., Papathanassiou, D., Crivello, F., Etard, O., Delcroix, N., et al. (2002). Automated anatomical labeling of activations in SPM using a macroscopic anatomical parcellation of the MNI MRI single-subject brain. Neuroimage 15, 273-289. doi: 10.1006/nimg.2001.0978

van den Heuvel, M. P., Mandl, R. C., Stam, C. J., Kahn, R. S., and Pol, H. E. H. (2010). Aberrant frontal and temporal complex network structure in schizophrenia: a graph theoretical analysis. J. Neurosci. 30, 15915-15926. doi: 10.1523/JNEUROSCI.2874-10.2010

Vawter, M. P., Evans, S., Choudary, P., Tomita, H., Meador-Woodruff, J., Molnar, M., et al. (2004). Gender-specific gene expression in post-mortem human brain: localization to sex chromosomes. Neuropsychopharmacology 29, 373. doi: 10.1038/sj.npp.1300337

White, T., van der Ende, J., and Nichols, T. E. (2019). Beyond bonferroni revisited: concerns over inflated false positive research findings in the fields of conservation genetics, biology, and medicine. Conserv. Genet. 20, 927-937. doi: 10.1007/s10592-019-01178-0

Yu, M., Sporns, O., and Saykin, A. J. (2021). The human connectome in alzheimer disease-relationship to biomarkers and genetics. Nat. Rev. Neurol. 17, 545-563. doi: 10.1038/s41582-021-00529-1

Conflict of Interest: The authors declare that the research was conducted in the absence of any commercial or financial relationships that could be construed as a potential conflict of interest.

Publisher's Note: All claims expressed in this article are solely those of the authors and do not necessarily represent those of their affiliated organizations, or those of the publisher, the editors and the reviewers. Any product that may be evaluated in this article, or claim that may be made by its manufacturer, is not guaranteed or endorsed by the publisher.

Copyright (ㄷ) 2021 Elsheikh, Chimusa, Alzheimer's Disease Neuroimaging Initiative, Mulder and Crimi. This is an open-access article distributed under the terms of the Creative Commons Attribution License (CC BY). The use, distribution or reproduction in other forums is permitted, provided the original author(s) and the copyright owner(s) are credited and that the original publication in this journal is cited, in accordance with accepted academic practice. No use, distribution or reproduction is permitted which does not comply with these terms. 